\title{
PROPHYLAXIS AGAINST ANTHRAX
}

\author{
Miroslav Špliňo ${ }^{1}$, Jiř̌ Patočka ${ }^{2}$ \\ Purkyně Military Medical Academy in Hradec Králové: Department of Epidemiology ${ }^{1}$, Department of Toxicology ${ }^{2}$
}

Summary: The paper presents fundamental knowledge concerning Bacillus anthracis and its potential terrorist misuse. The basic clinical forms are resumed with emphasis on inhalation infection from inspiration of B. anthracis spores. The AVA vaccine licensed in the United States, primary vaccination, protective efficacy of the vaccine, and adverse events are characterised. Stress is laid on pre-exposure and post-exposure prophylaxis of anthrax.

Key words: Anthrax; Inhalation anthrax; Bioterrorism; Spore-forming bacterium Bacillus anthracis; Vaccination; Chemoprophylaxis; Post-exposure prophylaxis

\section{Introduction}

In our previous paper (Acta Medica) we characterized the complex of Bacillus anthracis toxins. This knowledge is very important for solving an effective protection against anthrax infection. Chemoprophylaxis and vaccination play an important role in the system of pre-exposure and post-exposure prophylaxis against anthrax. Antimicrobial prophylaxis to prevent inhalation anthrax has been recommended for persons potentially exposed to Bacillus anthracis as a result of the recent bioterrorist attacks.

\section{Anthrax characterization}

Anthrax is a primary zoonosis. The disease is caused by spores of Bacillus anthracis. The disease affects both farm and wild animals (sheep, goats, cattle, antelopes, and other ruminants). Natural outbreaks of anthrax happened after a rainy period then and when cold weather arrives. In animal contamination is mainly digestive after consumption of plants or the carcasses of diseased animals. Persons become sick three ways, depending on the route of exposure: cutaneous, gastrointestinal, and inhalation. The infection occurs after a close contact with the infected animals, their tissues, when a direct exposure to $B$. anthracis occurs.

The cutaneous form develops after a contact of the skin with infected meat, wool or skin of the infected animals (skin cut or abrasion). In more than $95 \%$ cases anthrax manifests as cutaneous form. Skin infection begins as small papulae which progress to vesicles in 1-2 days followed by necrotic ulcer. Lesions are usually painless, patient may have fever, malaise, and regional lymphadenopathy. About $20 \%$ of untreated cases result in death, but seldom at an appropriate antimicrobial therapy.
The gastrointestinal form of anthrax: the incubation period 1-7 days. The disease is associated with a severe abdominal distress followed by fever, sign of septicemia. Characterised by acute inflammation of the intestinal tract, sore throat, disphagia, fever, vomiting blood. It results in death in $25-60 \%$ cases.

Inhalation anthrax is the most lethal form. Incubation period results from inhaling spores for 1-7 days, possibly up to 60 days. The pulmonary anthrax develops after inhalation of a spore aerosol, mainly at industrial processing of a contaminated wool, hair or hides. The initial symptom may resemble common cold: sore throat, fever, muscleaches. Symptoms progress after several days to severe breathing distress and shock. A direct transmission of pulmonary anthrax from man to man has not been proved. At the beginning of the 1950s an offensive research of B. anthracis was conducted together with preparation of possible use of anthrax spores as a biological weapon for its specific biological properties:

- Ability of spores to be transmitted by the respiratory route - aerosol

- High case-fatality rate

- Relatively easy preparation of the biological agent and surface distribution

- Ability for long-term storage with preservation of the full virulence for the spores (7)

\section{Anthrax and terrorism}

A possibility for terroristic anthrax spores misuse presupposes deliberate primary or secondary aerosolization of B. anthracis spores. Secondary aerosolization results from agitation of the settled spores, e.g. by contaminated dust, human activities handling animals, etc. The inhalation dose 
for anthrax spores in humans is not precisely known. The estimated infectious dose by the respiratory route required to cause inhalation anthrax in humans is $8,000-50,000$ spores based on data from studies of primates (11). The aerosol particles larger than 5 microns quickly subside and form a possibility for secondary aerosol formation. Smaller aerosol particles (1-5 microns in diameter) behave as a gas and persist in the environment without settling. The reported incubation period for inhalation anthrax ranges from 1 to 43 days (13).

An outbreak of inhalation anthrax occurred in the former Soviet Union in 1979. It was reported that the first clinical symptoms appeared up to 43 days after initial exposure. The exact date of exposure was only estimated and never exactly confirmed. The modal incubation period in that epidemic was reported as 9-10 days (1).

The incubation period after inhalation of $B$. anthracis spores is related to the dose. A post-exposure prophylaxis during the epidemic may prolong the incubation period. The experimental results of laboratory animals suggest that $B$. anthracis spores convert into a vegetative form in the host for several weeks post-infection. The inhaled spores in the alveolar spaces of the lungs are phagocyted by pulmonary macrophages. They gradually germinate and proliferate, multiply. A prophylactic administration of antibiotics doesn_t affect the germinating spores but only the vegetative germs of $B$. anthracis. After discontinuation of antibiotics the non-germinated spores (if sufficiently numerous) survive, new germination occurs and gradual impairment of the immune mechanisms appears and anthrax develops. The lethality in an untreated inhalation anthrax reaches 86-97\%.

In a 2001 terrorist misuse of anthrax in the USA the first inhalation form case was reported on $4^{\text {th }}$ October, 2001 and the last one on $31^{\text {st }}$ October, 2001. There were confirmed altogether 5 cases of the cutaneous form, 5 suspect forms, 9 cases of the inhalation anthrax, and 1 suspect form. Altogether 20 cases and 5 deaths form the inhalation anthrax (Department of Health and Human Services, USA - December, 2001). The development of the cutaneous and inhalation forms occurred after the skin contact of the post employees with a contaminated surface of the postal bags - sorting the letters, or a secondary whirl of a contaminated dust (inhalation of spores).

\section{Control and prevention of anthrax in animals}

Worldwide, anthrax among livestock is controlled through vaccination programs. A live unencapsulated variant of $B$. anthracis, strain Stern, has been used in the veterinary practice to protect livestock since 1939 (18). In case of death from anthrax the affected animals are buried or disposed of by burning. In prevention of human infection the following can be applied: veterinary control measures in breeding animals and slaughter-houses, and restriction of importation of hides and wool from epizootic regions of anthrax. It was repeatedly confirmed that injections of sterilised edema fluid from anthrax lesions provided a protective effect in laboratory animals. Nevertheless, even after inoculation of animals anthrax may develop. The induced immunity is short-term, it persists for 1-2 years.

\section{Protective immunity}

The first vaccine for humans (acellular vaccine) was developed in 1954. It was prepared from an aerobic $B$. anthracis culture filtrate adsorbed on aluminium potassium sulfate (19). The vaccine was tested on a monkey model. It exhibited low reactivity and short-term adverse events. During 1957-1960 the vaccine was improved through the selection of a suitable production $B$. anthracis strain with a high production a protective antigen and the use of aluminium hydroxide as the adjuvant (16). The vaccine was approved for use in the United States as anthrax vaccine adsorbed (AVA) (Patent number: 3,208,909 / 1965). Specific correlates for immunity against anthrax have not been identified. It is suggested that a humoral and cellular response against protective $B$. anthracis antigen (PA) is critical to protection against disease.

\section{Vaccine characterisation}

AVA is the anthrax vaccine licensed for the United States (FDA USA), produced by BioPort Corporation in Lansing, Michigan. The production strain is toxigenic and nonencapsulated - known as V 770-NP1-R (17). It is prepared from a cell-free filtrate of $B$. anthracis culture that contains no dead or live bacteria. The filtrate is adsorbed on to aluminium hydroxide adjuvant (Amphogel, Wyeth Laboratories), and contains all three toxin components: protective antigen (PA), lethal factor (LF) and oedema forming protein $(\mathrm{EF})$.

The vaccine AVP (Anthrax Vaccine Precipitated) is licensed in Great Britain, which contains protective antigen PA and lethal factor LF.

In the Czech Republic is not any licensed anthrax vaccine.

\section{Primary vaccination}

Primary vaccination consists of three subcutaneous injections at 0, 2 and 4 weeks, and three booster vaccinations at 6,12 , and 18 months. To maintain immunity, the manufacturer recommends an annual booster injection. The basis for the schedule of vaccinations is not well defined (5). Mild local reactions occur after vaccination: erythema, oedema, and induration smaller than $30 \mathrm{~mm}$ occurred after $20 \%$ of vaccinations. Moderate local reactions - defined as oedema and induration of $30-120 \mathrm{~mm}$ occurred after $3 \%$ vaccinations (2). Sever local reactions occurred after $1 \%$ of vaccinations. 
Systemic reaction occurred sporadically and have been reported approximately on the same scale after intramuscular and subcutaneous vaccine administration. Interruption of the vaccination schedule does not require restarting the entire series of anthrax vaccine or the addition of extra doses. No data have been published regarding use of anthrax vaccine among pregnant and breastfeeding women. The vaccine is contraindicated in persons who have recovered from anthrax (high percentage of adverse events).

Immunogenicity of a vaccine with reduced number of doses using intramuscular route of administration has been tested at present.

A published follow-up study of laboratory workers at Fort Detrick, concluded that, during the 25 year period following receipt of anthrax vaccine, the workers did not develop any unusual illness or unexplained symptoms associated with vaccination (14). In attempts to explain Persian Gulf War (PGW) syndrome the deployed veterans were examined who were vaccinated for anthrax. Existing scientific evidence does not support an association between anthrax vaccine and PGW illnesses (10).

\section{Vaccine efficacy}

The vaccine immunogenicity was tested in a clinical study with adult volunteers. Indirect hemagglutination was used to determine antibodies. Seroconversion 2 weeks after the first dose reached $83 \%$ and $91 \%$ after the second or more doses. Approximately $95 \%$ of vaccinees seroconvert with a fourfold rise in anti-PA IgG titres already after three doses. But the precise correlation between antibody titre and protection against infection is not defined (21). Data from the macaque model (Rhesus monkeys) documents that after administration of AVA vaccine the protective immunity is induced that protects monkeys against inhalation limiting dose of $B$. anthracis spores (11).

Preparation of new vaccines has been included in the research programme:

- Recombinant live vaccine: vaccine PA + Salmonella PA protective antigen

- Recombinant subunit vaccine: contains protective antigen which is expressed by Bacillus subtilis when using various adjuvants (Saponin, AL3+)

- $\left[4^{\text {th }}\right.$ Anthrax Conference, Annapolis, MD, June 2001 - Helene van Cuyck]

\section{Pre-exposure vaccination - AVA vaccine}

\section{Occupationally and laboratory engaged persons}

Routine vaccination is indicated for persons engaged in work involving production quantities or concentrations of B. anthracis cultures, and for persons in whom it is impossible to eliminate risk of the inhalation infection. The persons working at risk of a high contamination by anthrax from animal products - furs, bone meal, wool (3). Routine vaccination of veterinarians is not recommended in most countries. The limiting factor is incidence of anthrax in animals and a calculable risk assessment.

\section{Bioterroristic misuse of anthrax}

Preventive vaccination of the population, health-workers is nor recommended. Pre-exposure vaccination should be based on a calculable risk assessment but it cannot be predetermined objectively. Pre-exposure vaccination is considered in special military units and other select populations groups for which a calculable risk factor can be assessed (4).

\section{Post-exposure prophylaxis - chemoprophylaxis and vaccination}

Ciprofloxacin or doxycycline is recommended for antimicrobial prophylaxis and treatment of adults and children with $B$. anthracis infection associated with the recent bioterrorist attacks in the United State.

- Ciprofloxacin and ofloxacin are recommended for postexposure prophylaxis of anthrax based on assessment of in vitro susceptibility (minimum inhibitory concentrations - MIC: for ciprofloxacin and tetracycline $<0.06$ $\mathrm{microg} / \mathrm{ml}$ - susceptible; for amoxicillin $<0.03 \mathrm{microg} / \mathrm{ml}$ - susceptible) $(6,7)$. Post-exposure chemoprophylaxis with antibiotics was effective in animal model experiments. The duration of antibiotic therapy has not been determined exactly. Several studies have demonstrated that short-term administration of antibiotics (5-10 days) is not effective at preventing inhalation anthrax when large numbers of spores are inhaled (9).

By November, 2000 no resistance of naturally occurring anthrax strains to ciprofloxacin and tetracycline was reported (13). Antibiotics are effective against the germinated form of $B$. anthracis but are not effective against spores resident in the organism after an inhalation exposure spores may persist in the lung tissue without germination for months, as it was confirmed in experiments with monkeys (8). The length of persistence of $B$. anthracis spores in the lung tissue of exposed persons is not known.

Post-exposure prophylaxis against anthrax using chemoprophylactics is recommended after detectable or highly probable exposure to $B$. anthracis spores. Such exposure may only occur following a biological terrorist incident or laboratory accident. At the bioterrorism attack in New York in October, 2001 the recommended chemoprophylaxis was as follows:

- Ciprofloxacin at the dose of $500 \mathrm{mg}$, perorally, 2x daily for 60 days

- Doxycycline at the dose of $100 \mathrm{mg}$, perorally, 2x daily for 60 days

- Amoxicillin $500 \mathrm{mg}$ perorally, 3x daily for 60 days (prophylaxis in children)

The strain of $B$. anthracis used in the bioterrorist attack in NewYork was highly susceptible to ciprofloxacin and doxycycline, and was used for post-exposure prophylaxis in 
the inhalation and cutaneous forms of anthrax. The strain was also susceptible to chloramphenicol, clindamycin, rifampin, vancomycin, and clarithromycin. Combination of two antibiotics was recommended for the most severe forms of inhalation anthrax.

\section{Literatura}

1. Brachman PS, Plotkin SA, Bumford FH, Atchison MM. An epidemic of inhalation anthrax: the firs in the twentieth century. II. Epidemiology. Am J Hyg 1960;72:6-23.

2. Brachman PS, Gold H, Plotkin SA, Fekety FR, Werrin M, Ingraham NR. Field evaluation of a human anthrax vaccine. Am J Public Health 1962;52:632-45.

3. Brachman PS, Friedlander AM. Anthrax. In: Plotkin SA, Mortimer EA, eds. Vaccines. 2nd ed. Philadelphia, PA: WB Saunders Company, 1994:729-39.

4. CDC. Bioterrorism alleging use of anthrax and interim guidelines for management - US, 1998, MMWR 1999;48:69-74.

5. Darlow HM, Belton FC, Henderson DW. The use of anthrax antigen to immuniDarlow HM, Belton FC, Henderson DW. The use of ant
se man and monkey. Lancet 1956;476(September 8):9.

6. Doanay M, Aydin N. Antimicrobial susceptibility of Bacillus anthracis. Scand J Infect Dis 1991:23:333-5.

7. Franz DR, Jahrling PB, Friedlander AM, et al. Clinical recognition and management of patients exposed to biological warfare agents. JAMA 1997;278:399-411.

8. Friedlander AM, Welkos SL, Pitt MLM, et al. Postexposure prophylaxis against experimental inhalation anthrax. J Infect Dis 1993;167:1239-42.

9. Henderson DW, Peacock S, Belton FC. Observations on the prophylaxis of experimental pulmonary anthrax in the monkey. J Hyg 1956;54:28-36.

10. Iowa Persian Gulf Study Group. Self-reported illness and health status among Gulf War veterans: a population-based study. JAMA 1997;277:238-45.

11. Ivins BE, Fellowx PF, Pitt MLM, et al. Efficacy of a standard human anthrax vaccine against Bacillus anthracis aerosol spore challenge in rhesus monkeys. Salisbury Medical Bulletin (September 19 -21) 1995;87(suppl.):125-6.

12. Jemski JV. Respiratory virulence of Pasteurella tularensis Schu S4 strain, for man, monkey and guinea pig. April 15, 1963. DTIC recovery no. AD 498-288.
13. Lightfoot NF, Scott RJD, Turnbull PCB. Antimicrobial susceptibility of Bacillus anthracis. Salisbury Med Bull (April 11-13) 1990;68(suppl):95-8

14. Meselson M, Guillemin J, Hugh-Jones M, et al. The Sverdlovsk anthrax outhbreak of 1979. Science 1994;266:1202-7.

15. Peeler RN, Kadul PJ, Cluff LE. Intensive immunization of man: evaluation of possible adverse consequences. Ann Intern Med 1965;63:44-57.

16. ProMED-mail post. Anthrax, Human - USA: antibicrobial susceptibility, October 22, 2001.

17. Puziss M, Wright GG. Studies on immunity in anthrax. X. Gel-adsorbed protective antigen for immunization in man. J Bacteriol 1963;85:230-6.

18. Puziss M, Manning LC, Lynch JW, Barclay E, Abelow I, Whright GG. Large-scale production of protective antigen of Bacillus anthracis in aerobic culturec. Appl Microbiol 1963;11:330-4

19. Sterne M. The use of anthrax vaccines prepared from avirulent (unencapsulated) variants of Bacillus anthracis. Onderstepoort J Vet Sci An Ind 1939:13:307-12.

20. Tresselt HB, Boor AK. An antigen prepared in vitro effective for immunization against anthrax. III. Immunisation of monkeys against anthrax. J Infect Dis 1954;73:387-91.

21. Turnbull PCB, Broster MG, Carman JA, Manchee RJ, Melling J. Development of antibodies to protective antigen and lethal factor components of anthrax toxin in humans and guinea pigs and their relevance to protective immunity. Infect Immun 1986;52:356-63.

Submitted April 2002.

Accepted July 2002.

Prof. MUDr. Miroslav Špliňo, DrSc., Purkyně Military Medical Academy, Department of Epidemiology, Třebešská 1575, 50001 Hradec Králové, Czech Republic. e-mail: splino@pmfhk.cz 